\title{
Circle rail transit line timetable scheduling using Rail TPM
}

\author{
J. Zhibin, G. Jia \& X. Ruihua \\ School of Transportation Engineering, Tongji University, China
}

\begin{abstract}
Timetable scheduling is an important and difficult task in operation. Rail TPM is a user-friendly rail transit timetable scheduling tool, it can be easily scheduled for simple-path, share-path or circle-path trains. This paper emphasizes the definition of circle line topology structure, time-space structure, calculation and application of rolling stocks, rolling stocks assignment, train storage management, transfer schemes and so on. Finally, a case study of Line 4 in Shanghai illustrates the practical value of the Rail TPM program.
\end{abstract}

Keywords: rail transit, time-space diagram, circle routing, computing, Rail TPM.

\section{Introduction}

The growing mobility in the big cities of China puts pressure on both the road and the rail transit network. High quality rail transit services are needed to facilitate the increasing numbers of passengers. The rail transit system plays a key role in mobility in urban cities. With the rapid development of rail transit lines in China, more and more types of transit lines are in operation, such as "Y" type and circle type lines.

Rail transit has the characteristics of simple track, small train interval, flexible rolling stock turn-back, and high peak-time passenger flow and so on. Timetable scheduling is an important and difficult task in operation. The circle line is very important in the rail transit network. The flow characteristics, traffic organization and passenger organization of the circle line are different from other rail line types. In particular, the train diagram compilation in a circle line needs to consider the line structure, flow characteristics, customer service and rolling stock assignment. References [4] and [5] studied the key issues of time-space 
scheduling in a single routing line in detail. This paper mainly researches the key issues of timetable scheduling in a circle line with the computer tool Rail TPM, focuses on the line topology structure, time-space structure, rolling stock assignment, rolling stock storage management and transfer scheme of the circle line.

\section{Circle line and ring routing in urban mass transit}

\subsection{The characteristics of ring routing}

If the rail transit network has a circle line, the ring routing needs to be considered. In the circle routing, the trains always run in a certain direction (clockwise or counterclockwise); train turn-back operations are not needed. In some complicated rail transit networks, there are some special ring routings constituted by different lines, which are illustrated in fig. 1 .

\subsubsection{Single ring line}

The single ring line is commonly found in urban centers, showed in fig.1-a. It has the characteristics of small intervals, short distance stations, high peak-time passenger flow and high transfer passenger flow. This line type is common in developed rail transit networks, such as the Beijing metro line 2 and the Moscow circle metro line.

\subsubsection{Ring line plus straight line}

The straight line plus ring line is a special line kind, showed in fig.1-b and fig.1-c. The ring line and branch line can be connected with each other. The operational schemes of this line kind are complicated, and the time-space scheduling is more difficult. This line type is more common in a developed urban mass transit network, such as the Shanghai No. 3 line and No. 4 line (ring in the middle, there are nine shared stations), the Tokyo Yamanote Line (ring at the end) and Seoul Subway No. 2 line (one ring plus two branch lines).

\subsection{The basic characteristics of train operation in a circle line}

The train operation of a circle line has some significant features.

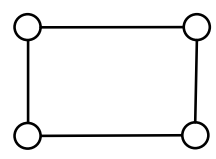

a. Single ring Line

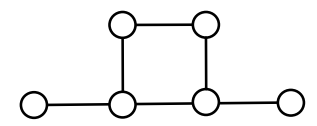

b. Ring in the middle

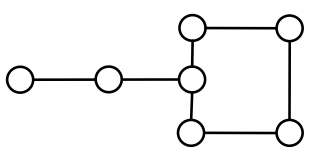

c. Ring in the end

Figure 1: Circle line structure in a rail transit network. 


\subsubsection{The turnaround of rolling stock is simple}

The ring line is linked together from beginning to end, the trains always run in a certain direction (clockwise or counterclockwise). The turn-back of rolling stock is very simple. There are no any fixed beginning stations and terminal stations, and there also are no train turnaround operations; in addition, the train operations can be out of or in depot.

\subsubsection{The operations of the inner ring and outer ring are independent,} which is suitable for the design of an asymmetric time-space diagram

The inner ring and outer ring are independent, so their operations will not interference each other, and the running intervals of the inner ring and outer ring could be different. These characteristics are beneficial for timetable scheduling, and the train operation adjustment is easier. For example, if the trains of one direction were delayed, the opposite direction's trains will not be influenced. That means the anti-interference capability of the circle line is more stable than the straight line.

\subsubsection{Different direction trains can reach the same destinations, and passengers' choices are flexible}

In the circle line, the trains in both directions could reach passengers' destinations, although the travel times are different. Regardless of the travel time, passengers could choose the comfortable direction to travel. In this way, the carrying capacity of both lines could be utilized fully to reduce the unbalanced degrees in different directions.

\section{Key issues of timetable scheduling in a circle transit line}

\subsection{Topology structure definition of a circle line}

With the strong connectivity, the topology structure of ring line should be defined firstly. For describing the characteristics of train operations in ring line, a station should be virtualized, then the ring line be virtualized to be a straight line. For example, the station "a" in fig. 2 was virtualized to be the stations "a" and "a".

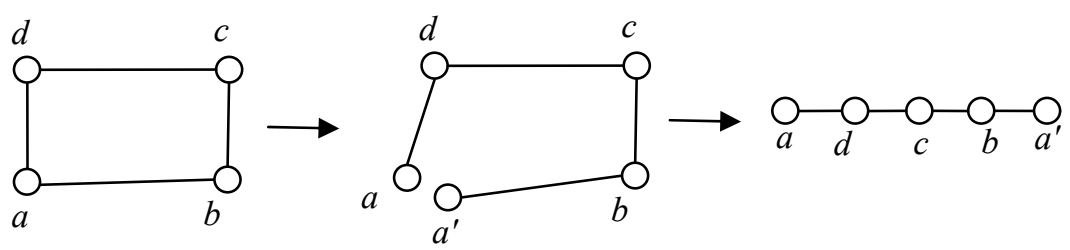

Figure 2: Topology structure of a circle line. 

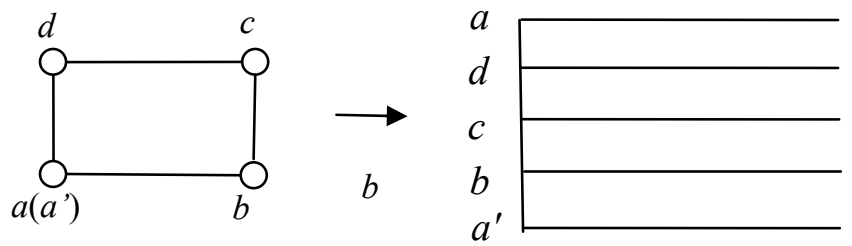

Figure 3: $\quad$ Time-space diagram structure of a circle line.

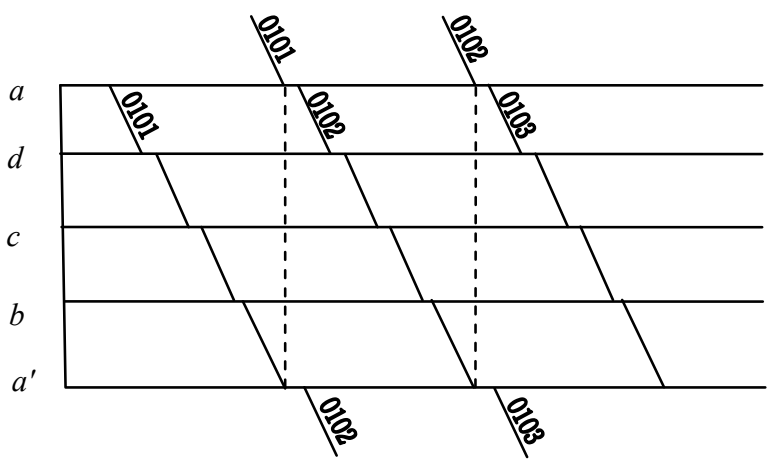

Figure 4: Time-space diagram of a circle line.

\subsection{The definition of the time-space diagram map structure}

Because of the complication of base map structure, the time-space diagram map structure of total line should be illustrated firstly for describing the train running. Reasonable base map structure is benefit to the rolling stock circulation. Fig. 3 is the example of time-space diagram structure.

\subsection{Display method of a running line}

In order to express train running process more clearly and directly, the display method of running line must be simply, directly and friendly, such as that showed in fig. 4.

\subsection{Calculation and application of rolling stocks}

In circle line, if the virtual original or terminal station only has double track, the trains could not stay in these stations too long. If no such, the follow-up trains will be affected. When scheduling in circle rail transit line, the running intervals and total running time should be considered synthetically.

The trains in inner ring and outer ring could run independently, so the number of rolling stocks needs to be calculated separately. The total number of rolling 
stocks is decided by the train interval and total running time. The computational formulas are as follows:

$$
\begin{gathered}
N=N^{\text {inner }}+N^{\text {outer }}+n_{\text {reserve }} \\
N^{\text {inner }}=\left\lceil T^{\text {inner }} / t_{\text {interval }}^{\text {inner }}\right] \\
N^{\text {outer }}=\left\lceil T^{\text {outer }} / t_{\text {interval }}^{\text {outer }}\right] \\
T^{\text {inner }}=\sum_{j=1}^{n-1} r_{n}^{\text {inner }}+\sum_{j=1}^{n} t_{n}^{\text {inner }} \\
T^{\text {outer }}=\sum_{j=1}^{n-1} r_{n}^{\text {outer }}+\sum_{j=1}^{n} t_{n}^{\text {outer }}
\end{gathered}
$$

where $\mathrm{N}$ is the number of rolling stocks; $N^{\text {inner }}$ is the number of rolling stocks in inner circle ring (unit); $N^{\text {outer }}$ is the number of rolling stocks in outer circle ring (unit); $n_{\text {reserve }}$ is the number of reserve rolling stocks (unit); $T^{\text {inner }}$ is the cycle time in inner ring (s); $T^{\text {outer }}$ is the cycle time in outer ring $(\mathrm{s}) ; t_{\text {interval }}$ is the average interval; $n$ is the total number of stations; $r$ is the running time (s); $t$ is the stopping time (s).

\subsection{Rolling stock storage management}

If the ring lines have many depots, there will be various scheme of rolling stock storage management. The rolling stock operation of depot should consider the flexibility and economy firstly.

\subsection{Schedule of first and last trains}

The management of first and last trains is an important factor of scheduling. Circle line has many transfer nodes, so the running operation of first and last trains in other lines should consider the schedule of circle line.

\subsection{Connection of trains in the transfer station}

The connection of trains in transfer station should to be considered emphatically for shortening the transfer time of passengers.

\section{Timetable scheduling process in a circle routing line}

When computing the time-space diagram in circle routing, the line topology structure and base map structure should be constructed firstly. Based on the passenger flow and running intervals, the total running cycle time in inner ring and outer ring should be calculated separately. After matching the running 
intervals and running cycles, the number of rolling stocks could be calculated to compile the time-space diagram. Then the rolling stock storage management and transfer scheme of circle line could be made out. At last, the time-space graph and relevant indexes can be exported. The total process is illustrated in fig. 5 .

\section{Case application}

The above designing thought has been applied to the Rail Transit Train Plan Maker System (Rail TPM V4.8). Rail TPM is a user-friendly rail transit timetable scheduling tool, it can be easily scheduled the simple-path, share-path or circle-path trains. Using this software, the time-space diagrams of Shanghai Metro Line 4 and Beijing Metro Line 2 had been compiled successfully. With this tool, the efficiency and speed of computing time-space diagrams could be raised.

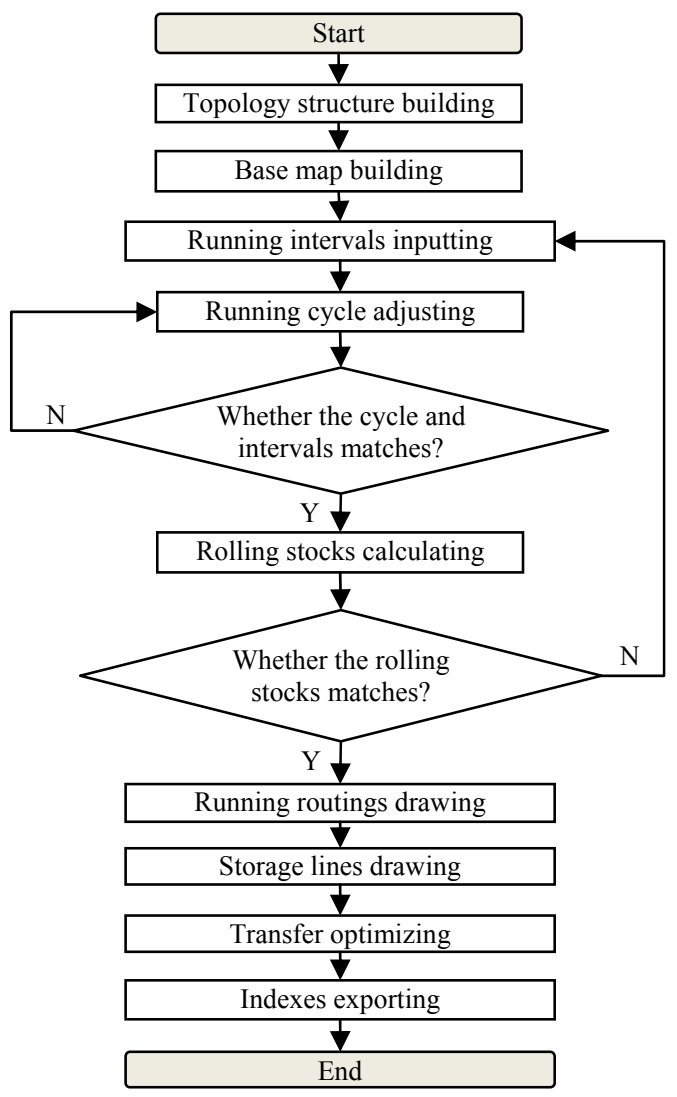

Figure 5: Flowchart of time-space diagram computing in circle routing. 


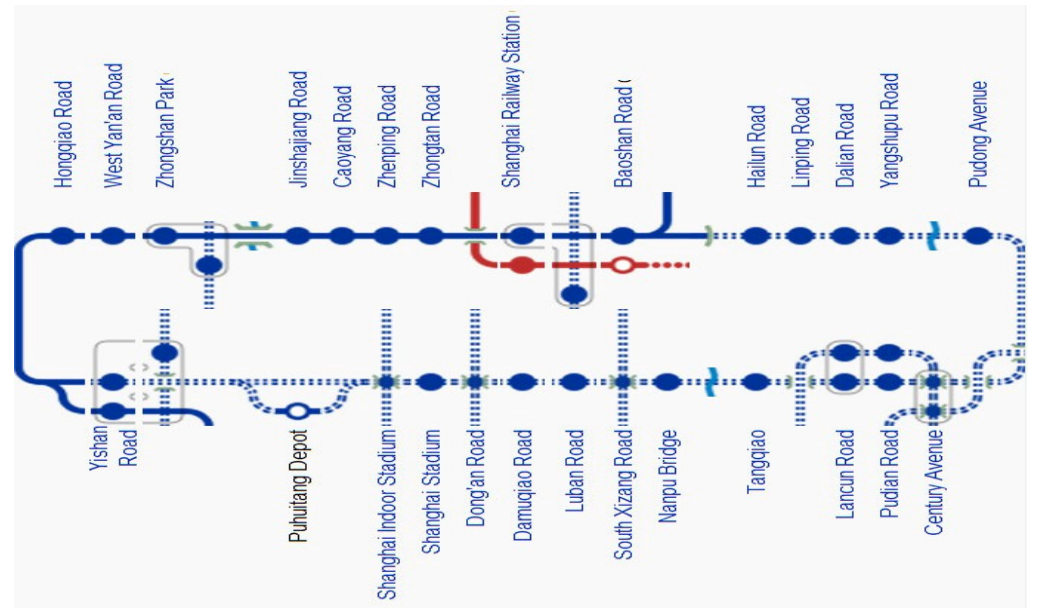

Figure 6: $\quad$ Metro line 4 in Shanghai.

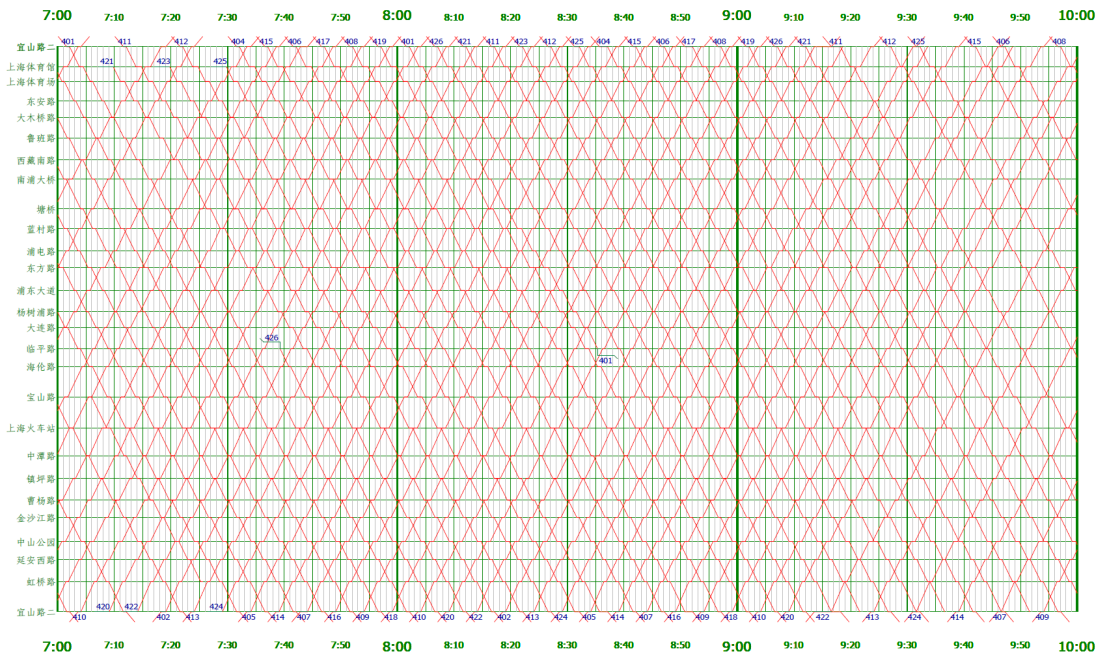

Figure 7: $\quad$ 409\# time-space diagram in Shanghai Metro line 4.

The Shanghai Metro Line 4 is about 34 kilometers, with 26 stations, and has nine shared stations with metro Line 3 (showed in fig. 6). Fig. 7 is the time-space diagram examples of Metro Line 4.

\section{Conclusion}

To computing the time-space diagram in circle lines, the line topology structure, time-space structure, rolling stock assignment, rolling stock storage management and transfer scheme should be considered comprehensively. With the frequently 
change of passenger flow characteristics in time and space, the time-space diagrams in circle line could be computed with Rail TPM timely. The computing efficiency and passenger service level could be raised.

\section{References}

[1] Wong Rachel, C. W., Yuen Tony, W. Y., Fung Kwok Wah et al. Optimizing timetable synchronization for rail mass transit. Transportation Science, 42(1), pp.57-69, 2008.

[2] Peeters Marc \& Kroon Leo. Circulation of railway rolling stock: a branchand-price approach. Computers \& Operations Research, 35(2), pp.538-556, 2008.

[3] Kroon Leo, Maroti Gabor, Helmrich Mathijn Retel et al. Stochastic improvement of cyclic railway timetables. Transportation Research Part B: Methodological, 42(6), pp.553-570, 2008.

[4] Xu Ruihua, Jiang Zhibin. Key problems of designing train timetable in urban mass transit system with computer. Urban mass transit research, 8(5), pp.31-35, 2005.

[5] Jiang Zhibin, Xu Ruihua, Designing multi-interval train working diagram in urban mass transit system with computer. International Doctoral Student Innovation Forum in Traffic and Transportation Engineering, Zhu Zhaohong, China Communications Press: Beijing, pp.75-82, 2005. 